\title{
Gait Speed as a Screening Tool for Foot Pain and the Risk of Falls in Community-Dwelling Older Women: A Cross-Sectional Study
}

This article was published in the following Dove Press journal:

Clinical Interventions in Aging

\author{
Lovro Štefan (iD) \\ Mario Kasović ${ }^{1,2}$ \\ Martin Zvonar ${ }^{2,3}$ \\ 'Faculty of Kinesiology, Department of \\ General and Applied Kinesiology, \\ University of Zagreb, Zagreb, Croatia; \\ ${ }^{2}$ Faculty of Sports Studies, Department of \\ Sports Motorics, Masaryk University, \\ Brno, Czech Republic; ${ }^{3}$ RECETOX, \\ Faculty of Science, Masaryk University, \\ Brno, Czech Republic
}

Correspondence: Lovro Štefan Faculty of Kinesiology, Department of General and Applied Kinesiology, University of Zagreb, Horvaćanski Zavoj

15, Zagreb, Croatia

Tel +385-98-9177-060

Email lovro.stefan1510@gmail.com
Purpose: The main purpose of the study was to establish a gait speed cut-off value to predict foot pain and the risk of falls among community-dwelling older adults.

Patients and Methods: In this cross-sectional study, one-hundred and twenty White older women speaking Croatian (mean \pm SD age $71.02 \pm 6.78$ years, height $161.77 \pm 6.23 \mathrm{~cm}$, weight $70.29 \pm 12.97 \mathrm{~kg}$, body mass index $26.79 \pm 4.42 \mathrm{~kg} / \mathrm{m}^{2}$ ) were recruited. The prevalence of foot pain was assessed by a single-item question and the risk of falls by the Downtown Fall Risk Index with a proposed cut-off value of "low risk" ( $<3$ points) vs "high risk" ( $\geq 3$ points) of falls. Self-selected gait speed (the independent variable) was estimated with a pressure platform (Zebris Company, Munich, Germany).

Results: Mean gait speed was $0.95 \mathrm{~m} / \mathrm{s}$. Of the total sample, $53.30 \%$ and $33.30 \%$ reported foot pain and had higher risk of falls. For foot pain and the risk of falls, gait speed cut-off values were $0.88 \mathrm{~m} / \mathrm{s}$ and $0.85 \mathrm{~m} / \mathrm{s}$ (area under the curve $=0.80$ and 0.83 , standard error $=$ 0.043 and $0.043, p<0.001$ ). Sensitivity for foot pain and the risk of falls was $66.20 \%$ and $85.90 \%$ and specificity was $84.80 \%$ and $69.00 \%$. Slower gait speed was associated with higher prevalence of foot pain $(\mathrm{OR}=10.92,95 \% \mathrm{CI} 4.28$ to $27.89, p<0.001)$ and higher risk of falls (OR $=13.59,95 \%$ CI 5.45 to $33.87, p<0.001)$.

Conclusion: Proposed gait speed values of $0.88 \mathrm{~m} / \mathrm{s}$ and $0.85 \mathrm{~m} / \mathrm{s}$ may be used in clinical settings to predict foot pain and the risk of falls among community-dwelling older women.

Keywords: velocity, discomfort, elderly, risk, falls

\section{Introduction}

Foot problems are common in older people. ${ }^{1}$ The structure of the foot becomes more widened and flattened with the fat padding beneath the foot exhibiting greater stiffness. ${ }^{2}$ Among numerous risk factors associated with foot problems, ${ }^{1}$ foot pain represents a leading cause of mobility limitations and lower quality of life in older individuals. $^{3,4}$ It is estimated that approximately $1 / 4$ of adults suffer from foot pain, ${ }^{5}$ increasing the costs of health care service. ${ }^{6}$ Frequent foot pain also doubles the risk of falling, ${ }^{7}$ which often leads to early institutionalization in nursery homes and premature death. ${ }^{8}$ It has been well-documented that foot pain is a significant predictor of falls in older adults ${ }^{7,9,10}$ and these two factors often coexist. ${ }^{7}$

In older adults, the most common way to engage in regular physical activity is by walking. ${ }^{11}$ Walking speed significantly decreases by age, steadily affecting the gait biomechanics and leading to all-cause mortality. ${ }^{12}$ Previous evidence has shown that gait speed is a clinically meaningful tool for testing neuromotor control 
of mobility and overall physical performance ${ }^{6,13}$ Although the associations between gait speed, foot pain and the risk of falls have been well established, ${ }^{6,14-20}$ indicating that slower gait speed increases the aforementioned risks, little evidence has been provided regarding the establishment of relevant gait speed cut-off values to predict foot pain and the risk of falls. ${ }^{18,21,22}$ Specifically, a study by Beauchet et $\mathrm{al}^{21}$ has shown that gait speed cut-off values for those with no history, history and recurrent history of falls are documented as $0.75 \mathrm{~m} / \mathrm{s}, 0.73 \mathrm{~m} / \mathrm{s}$ and $0.59 \mathrm{~m} / \mathrm{s}$. Another study showed that gait speed $\leq 0.56 \mathrm{~m} / \mathrm{s}$ predicted falls in older men with both sensitivity and specificity of $70.00 \%{ }^{22}$ To the best of the authors' knowledge, no study to date has systematically established a cut-off value for gait speed in predicting foot pain. Despite the effort of using gait speed as a screening tool to predict the risk of falls, ${ }^{18,21,22}$ evidence suggests that the discrimination and predictive validity of gait speed in classifying older adults who are more prone to falling is yet to be established. ${ }^{18}$ Since both foot pain and falls are major risk factors for hospitalization and premature death in older adults, ${ }^{7,8}$ it is necessary to investigate screening properties of gait speed in detecting those individuals at higher risk of foot pain and the risk of falls.

Therefore, the main purpose of the study was to determine clinically significant gait speed cut-off values to predict foot pain and the risk of falls among communitydwelling older adults.

\section{Patients and Methods Study Participants}

In this cross-sectional study, we recruited older women from the city of Zagreb. More detailed description of the sample size, study methodology and inclusion criteria can be found elsewhere. ${ }^{23,24}$ The inclusion criteria were: 1) being $\geq 60$ years old; 2) living independently in the community; 3) passing the Short Portable Mental Status Questionnaire; 4) being able to ambulate for at least $10 \mathrm{~m}$ with or without an aid; 5) being free from neurological diseases; and 6) could arrange their own transport to a testing venue in their community. In brief, we approached five neighborhoods of estimated population of older adults aged $\geq 60$ years to be $\approx 1500$. We set the confidence level of $95 \%$ and marginal error of $10 \%$ to get an appropriate sample size of 110 . After spreading the main information about the project, 210 volunteers agreed to take part in the study. Of these, 17 could not be part of the study due to personal issues and 73 failed to provide full data. Finally, our analyses were based on 120 older women. Before the study began, all participants had given a written informed consent to participate and were able to leave the study on their own. All procedures performed in this study were anonymous (the participants only provided the data regarding basic sociodemographic characteristics and no names were recorded) and we followed the recommendations of Declaration of Helsinki. The Ethical Committee of the Faculty of Kinesiology, University of Zagreb, Croatia approved the study (Ethics code number: 2019).

\section{Foot Pain}

To assess the prevalence of foot pain, we asked the participants the following question: "On most days do you have pain, aching or stiffness in either of your feet" with the following responses: 1) "No"; 2) "Yes, left foot only"; 3) "Yes, right foot only"; 4) "Yes, both feet"; 5) "Yes, not sure what side"; and 6) "Unknown". ${ }^{25}$ The outcome measure was having "no foot pain" ("No" response) vs "foot pain" ("Yes, left foot only", "Yes, right foot only", "Yes, both feet" and "Yes, not sure what side"). None of the participants responded with "Unknown" answer.

\section{The Risk of Falls}

The risk of falls was estimated using the reliable and valid Downtown Fall Risk Index, ${ }^{26}$ a questionnaire consisting of five questions: 1) Known previous falls ("No", "Yes"); 2) Using medication ("None", "Tranquillizers/sedatives", "Diuretics", "Antihypertensives", "Antiparkinsonian drugs", "Antidepressants" and "Other medications"); 3) Having sensory deficits ("None", "Visual impairment", "Hearing impairment" and "Limb impairment"); 4) Mental state status ("Oriented" and "Confused"); and 5) Gait characteristics ("Normal", "Safe with walking aids", "Unsafe" and "Unable"). When completed, the final score ranges from 0 to 11 , where a higher score indicates a higher risk of falls. For the purpose of the study, the outcome measure was dichotomized as "low" risk $(<3$ points scored) vs "high risk" ( $\geq 3$ points scored). ${ }^{26}$ The agreement level between questions in the questionnaire was satisfactory (Cronbach's $\alpha=0.72$ ).

\section{Gait Speed}

To estimate gait speed, we used a pressure platform (Zebris Company, FDM, GmbH, Munich, Germany; number of sensors 11.264; sampling rate $100 \mathrm{~Hz}$; sensor area 
$149 \mathrm{~cm} \times 54.2 \mathrm{~cm})$. More detailed information about the protocol and data collection has been described previously. $^{23,24}$ In brief, the walkway was $10.5 \mathrm{~m}$ in length (1.5-m platform and 4.5-m custom-designed dense material before and after the platform). The protocol consisted of a set of instructions for participants to walk at a preferred speed and to look straight forward across the platform being barefoot and without targeting the platform. When the participants finished walking across a $10.5-\mathrm{m}$ walkway, they needed to turn around for $180^{\circ}$ and continued to walk again over the platform until they reach the end of the walkway. The aforementioned protocol was repeated once again with a total of 4 trials across the platform. Having 3-5 trials has shown greater reliability in non-healthy older adults. ${ }^{27}$ If we detected obvious gait deviations, we discarded the trials and we repeated the protocol. Finally, software generated spatial and temporal data, including gait speed in $\mathrm{m} / \mathrm{s}$.

\section{Data Analysis}

Basic descriptive statistics are presented as mean \pm SD for numerical variables and as frequency (\%) for nominal variables. The receiver operating curve was created using a non-parametrical Mann-Whitney method with the area under the curve value to determine the accuracy of gait speed to predict foot pain and the risk of falls. Of note, an area under the curve $\geq 0.5$ was considered to have diagnostic value. Of note, the outcome measures for foot pain and the risk of falls were having "no foot pain" ("No" response) vs "foot pain" ("Yes, left foot only", "Yes, right foot only", "Yes, both feet" and "Yes, not sure what side") and $<3$ points ("low risk") vs $\geq 3$ points scored on a scale ("high risk"). A set of cross-tabulation matrices were run to estimate sensitivity (the true positive rate) and specificity (the true negative rate) rates. The level of agreement between gait speed, foot pain and the risk of falls was calculated with kappa $(\kappa)$ statistics with the following magnitudes: 1) 0.00-0.20: none; 2) 0.21-0.39: minimal; 3) 0.40-0.59: fair; 4) 0.60-0.79: moderate; 5) 0.80-0.90: strong; and 6) >0.90: almost perfect. ${ }^{28}$ Logistic regression analyses were used to calculate the associations between newly proposed gait-speed cut-off values to predict the presence of foot pain and the risk of falls. The results of logistic regressions are presented as the odds ratio (OR) with $95 \%$ confidence interval $(95 \% \mathrm{CI})$. Of note, age was not significantly correlated with gait speed, foot pain and the risk of falls in our sample $(r=-0.17-0.09, p>0.05)$, so we dropped out age-specific analyses. All analyses were
Table I Basic Descriptive Statistics of the Study Participants $(\mathrm{N}=120)$

\begin{tabular}{|l|l|l|}
\hline Study Variables & Mean \pm SD & Min-Max \\
\hline Age (years) & $71.01 \pm 6.77$ & $60.00-86.00$ \\
Height $(\mathrm{m})$ & $161.78 \pm 6.22$ & $148.10-182.40$ \\
Weight $(\mathrm{kg})$ & $70.29 \pm 12.97$ & $47.00-114.00$ \\
Body mass index $\left(\mathrm{kg} / \mathrm{m}^{2}\right)$ & $26.79 \pm 4.42$ & $18.83-45.09$ \\
Gait speed (m/s) & $0.95 \pm 0.24$ & $0.39-1.50$ \\
Foot pain $(\%$ of "Yes")* & $53.30 \%$ & $/$ \\
The risk of falls $(\geq 3, \%)^{*}$ & $33.30 \%$ & $/$ \\
\hline
\end{tabular}

Notes: *Denotes using percentage (\%), $p \leq 0.05$.

calculated using the Statistical Packages for Social Sciences (SPSS Inc., Chicago, IL, USA) program with a statistical significance of $p \leq 0.05$.

\section{Results}

Basic descriptive statistics are presented in Table 1. Average gait speed was slightly below $1 \mathrm{~m} / \mathrm{s}$. More than half of the participants reported experiencing foot pain and approximately $1 / 3$ of them were at higher risk of falls.

Table 2 shows the receiver operating curve values for gait speed as the test variable and foot pain and the risk of falls as state variables. The gait speed cut-off values for foot pain and the risk of falls were $0.88 \mathrm{~m} / \mathrm{s}$ and $0.85 \mathrm{~m} / \mathrm{s}$.

Sensitivity and specificity values for the obtained gait speed cut-offs are presented in Table 3 (foot pain) and Table 4 (the risk of falls). Specifically, sensitivity and specificity of gait speed $\leq 88 \mathrm{~m} / \mathrm{s}$ to correctly predict foot pain were $66.20 \%$ and $84.80 \%$. Sensitivity and specificity of gait speed $\leq 85 \mathrm{~m} / \mathrm{s}$ to correctly predict the risk of falls were $85.90 \%$ and $69.00 \%$. The level of agreement between newly proposed gait speed cut-offs, foot pain and the risk of falls was fair $(\kappa=-0.49$ and $-0.45, p<0.001)$. Participants who walked $\leq 0.88 \mathrm{~m} / \mathrm{s}$ and $\leq 0.85 \mathrm{~m} / \mathrm{s}$ were almost 11 times more likely to report foot pain $(\mathrm{OR}=$ $10.92,95 \%$ CI 4.28 to $27.89, p<0.001$ ) and were approximately 13.5 times more likely to have higher risk of falls

Table 2 Area Under the Curve of Gait Speed Specified for Foot Pain and the Risk of Falls in the Study Participants $(N=120)$

\begin{tabular}{|c|c|c|c|c|}
\hline $\begin{array}{l}\text { Outcome } \\
\text { Variables }\end{array}$ & $\begin{array}{l}\text { Area } \\
\text { Under } \\
\text { the Curve }\end{array}$ & $\begin{array}{l}\text { Standard } \\
\text { Error* }\end{array}$ & $p$-value & $95 \% \mathrm{Cl}$ \\
\hline Foot pain & 0.80 & 0.043 & $<0.001$ & 0.72 to 0.88 \\
\hline $\begin{array}{l}\text { The risk of } \\
\text { falls }\end{array}$ & 0.83 & 0.043 & $<0.001$ & 0.75 to 0.91 \\
\hline
\end{tabular}

Notes: $*$ Denotes standard error under the non-parametric assumption, $p \leq 0.05$. 
Table 3 Cross-Tabulation Matrix of Gait Speed Cut-Off Value and Foot Pain in the Study Participants $(N=120)$

\begin{tabular}{|l|l|l|l|}
\hline \multirow{2}{*}{$\begin{array}{l}\text { Gait Speed Cut- } \\
\text { Off Value }\end{array}$} & \multicolumn{2}{|l|}{ Foot Pain } & \multirow{2}{*}{$\begin{array}{l}\text { K Statistics } \\
\text { ( } p \text {-value) }\end{array}$} \\
\cline { 2 - 3 } & $\begin{array}{l}\text { No Foot } \\
\text { Pain }\end{array}$ & $\begin{array}{l}\text { Foot } \\
\text { Pain }\end{array}$ & \\
\hline$\leq 0.88 \mathrm{~m} / \mathrm{s}$ & $7(15.20)^{*}$ & $49(66.20)$ & $-0.49(<0.00 \mathrm{I})$ \\
$>0.88 \mathrm{~m} / \mathrm{s}$ & $39(84.80)$ & $25(33.80)$ & \\
\hline
\end{tabular}

Notes: *Denotes using frequency and percentage (\%).

Table 4 Cross-Tabulation Matrix of Gait Speed Cut-Off Value and the Risk of Falls in the Study Participants $(N=120)$

\begin{tabular}{|l|l|l|l|}
\hline \multirow{2}{*}{$\begin{array}{l}\text { Gait Speed Cut- } \\
\text { Off Value }\end{array}$} & \multicolumn{2}{|l|}{ The Risk of Falls } & \multirow{2}{*}{$\begin{array}{l}\text { S Statistics } \\
\text { (p-value) }\end{array}$} \\
\cline { 2 - 3 } & $\begin{array}{l}\text { Low Risk } \\
(<3)\end{array}$ & $\begin{array}{l}\text { High Risk } \\
(\geq 3)\end{array}$ & \\
\hline$\leq 0.85 \mathrm{~m} / \mathrm{s}$ & $13(31.00) *$ & $67(85.90)$ & $-0.45(<0.00 \mathrm{I})$ \\
$>0.85 \mathrm{~m} / \mathrm{s}$ & $29(69.00)$ & II (14.10) & \\
\hline
\end{tabular}

Note: *Denotes using frequency and percentage (\%).

$(\mathrm{OR}=13.59,95 \%$ CI 5.45 to $33.87, p<0.001)$. Of note, when we put gait speed as a continuous variable, participants were 85.0 and 74.0 times more likely to suffer from foot pain and had higher risk of falls. Similar associations remained after adjusting for age and body mass index. Of note, the correlation between foot pain and the risk of falls was fair $(\kappa=0.35, p<0.001)$.

\section{Discussion}

The main purpose of the study was to determine clinically significant gait speed cut-off values to predict foot pain and the risk of falls among community-dwelling older adults.

Our main findings are: 1) gait speed cut-off values of $0.88 \mathrm{~m} / \mathrm{s}$ and $0.85 \mathrm{~m} / \mathrm{s}$ predict foot pain and the risk of falls; 2) those individuals who walk at $\leq 0.88 \mathrm{~m} / \mathrm{s}$ and $\leq 0.85 \mathrm{~m} / \mathrm{s}$ are more likely to report foot pain and be at higher risk of falls; and 3) the aforementioned gait speed cut-off values can predict $20.25 \%$ and $24.01 \%$ of foot pain and the risk of falls.

This is the first study defining specific gait speed cut-off values in predicting foot pain and the risk of falls in older women simultaneously. Previous studies have shown that gait speeds of $0.59 \mathrm{~m} / \mathrm{s}, 0.73 \mathrm{~m} / \mathrm{s}$ and $0.75 \mathrm{~m} / \mathrm{s}$ predict recurrent, history and no history of falls among older adults in residential homes. ${ }^{21}$ Another study conducted among older men showed that gait speed $\leq 0.56 \mathrm{~m} / \mathrm{s}$ predicted higher risk of falls. ${ }^{22}$ The discrepancy between the findings may be explained by different characteristics of the participants (living in residential homes and being $\geq 75$ years of age ${ }^{21}$ only male veterans included ${ }^{22}$ ), compared to freeliving older women in our study. Also, we assessed gait speed using a Zebris pressure platform, while other studies used a $10-\mathrm{m}$ straight walk test ${ }^{21}$ and permanent markers attached with masking tape to the back of the heel on a 6-m brown paper walkway. ${ }^{22}$ Finally, the risk of falls was assessed by the past year history of falls, ${ }^{21,22}$ while we used the Downtown Fall Risk Index questionnaire, ${ }^{26}$ which might have led to different fall perception and cutoff values. Other studies reported no cut-off values indicating higher risk of falls. ${ }^{18}$ The majority of the studies aiming to explore the associations between gait speed and the risk of falls have shown that slower gait speed $(<0.60 \mathrm{~m} / \mathrm{s})$ is associated with higher prevalence of falls, compared to intermediate $(0.60 \mathrm{~m} / \mathrm{s}-1.00 \mathrm{~m} / \mathrm{s})$, normal $(1.00 \mathrm{~m} / \mathrm{s}-$ $1.30 \mathrm{~m} / \mathrm{s})$ and fast $(>1.30 \mathrm{~m} / \mathrm{s})$ gait speed. ${ }^{18}$ However, another study showed a U-shaped association between gait speed and the risk of falls, pointing out that slow and fast performers were at higher risk of falls, compared to older adults with normal gait speed $(<1.3 \mathrm{~m} / \mathrm{s}) .{ }^{15}$ Of note, we classified our participants as being slow $(<0.60 \mathrm{~m} / \mathrm{s})$, intermediate/normal $(0.60 \mathrm{~m} / \mathrm{s}-1.30 \mathrm{~m} / \mathrm{s})$ and fast $(>1.30 \mathrm{~m} / \mathrm{s})$ walkers and showed that those individuals walking $<0.60 \mathrm{~m} / \mathrm{s}$ were 15 times more likely to have higher risk of falls (OR $=15.00,95 \%$ CI 1.79 to $125.62, p=0.013)$, while those being categorized as fast walkers were $73.9 \%$ less likely to have higher risk of falls $(\mathrm{OR}=0.26,95 \% \mathrm{CI}$ 0.10 to $0.66, p=0.004)$. This means that slow gait speed may be more important for screening the risk of falls, compared to fast gait speed.

Our study also shows that older women walking $\leq 0.88 \mathrm{~m} / \mathrm{s}$ are more likely to report foot problems, with sensitivity and specificity of $66.20 \%$ and $84.80 \%$. To the best of our knowledge, this is the first study establishing a cut-off value of gait speed to predict foot pain in older individuals. Evidence shows that pain may disrupt mobility ${ }^{29}$ and gait limitations, due to arthritic back and joint changes. ${ }^{30} \mathrm{~A}$ few previous studies have examined the association between pain and gait speed. ${ }^{6,31,32}$ In general, findings are consistent and show that both pain severity and multisite pain are associated with slow gait speed, even after adjusting for potential confounders. It has been documented that the most common location of foot pain is in the toe region, often associated with toe deformities (claw or hammer toe) and reported by almost $50.00 \%$ of older individuals. ${ }^{7}$ Since foot pain and the 
risk of falls often coexist, ${ }^{7}$ it is not surprising that slower gait speed is associated with both foot pain and the risk of falls in our study. Moreover, a new gait speed cut-off value of $\leq 0.88 \mathrm{~m} / \mathrm{s}$ can be used by health-related professionals as a diagnostic tool in detecting a "risky" group of older women, who may be more vulnerable for having foot pain.

This study is not without limitations. First, our analyses are based on a cross-sectional design, and causal associations cannot be drawn. Second, a relatively small sample might have underpowered the associations. Third, we based our findings on older women, so we cannot make the generalizability to men $\geq 60$ years of age. Fourth, we included apparently healthy older adults and we can only speculate that gait speed cut-off values may have been different in less healthy individuals. Finally, we did not collect the additional data regarding health conditions, which might have also led to different cut-off values and discriminative ability. Therefore, future studies aiming to determine optimal cut-off points should be populationbased in a followup design, in addition adjusted for potential confounding variables.

\section{Conclusions}

Our study shows that gait speed cut-off values of $0.88 \mathrm{~m} / \mathrm{s}$ and $0.85 \mathrm{~m} / \mathrm{s}$ significantly predict foot pain and the risk of falls in older women. Slower gait speed may contribute to foot pain and higher prevalence of falls. By using the proposed cut-off values, individuals who walk slower should be a target population for special interventions that improve foot biomechanics and reducing both foot pain and future risk of falls.

\section{Data Sharing Statement}

All the data can be assessed by reasonable request from the corresponding author.

\section{Ethics Approval and Consent to Participate}

Written informed consent. The Ethical Committee of the Faculty of Kinesiology, University of Zagreb, Croatia approved the study (Ethics code number: 2019). Consent for publication: Obtained.

\section{Acknowledgments}

We would like to thank all the participants for their enthusiastic participation in the study.

\section{Author Contributions}

All authors made substantial contributions to conception and design, acquisition of data, or analysis and interpretation of data; took part in drafting the article or revising it critically for important intellectual content; agreed to submit to the current journal; gave final approval of the version to be published; and agree to be accountable for all aspects of the work.

\section{Funding}

This paper was self-funded.

\section{Disclosure}

The authors report no conflicts of interest for this work.

\section{References}

1. Menz HB. Foot Problems in Older People: Assessment and Management. 1st ed. New York: Churchill Livingstone; 2008.

2. Menz HB. Biomechanics of the ageing foot and ankle: a mini review. Gerontology. 2015;61:381. doi:10.1159/000368357

3. Menz HB, Dufour AB, Casey VA, et al. Foot pain and mobility limitations in older adults: the Framingham Foot Study. J Gerontol A Biol Sci Med Sci. 2013;68:1281-1285. doi:10.10 93/gerona/glt048

4. Riskowski JL, Hagedorn TJ, Dufour AB, Hannan MT. Functional foot symmetry and its relation to lower extremity physical performance in older adults: the Framingham Foot Study. J Biomech. 2012;45:1796-1802. doi:10.1016/j.jbiomech.2012.04.019

5. Thomas MJ, Roddy E, Zhang W, Menz HB, Hannan MT, Peat GM. The population prevalence of foot and ankle pain in middle and old age: a systematic review. Pain. 2011;152:2870-2880. doi:10.1016/j. pain.2011.09.019

6. Ogawa EF, Shi L, Bean JF, et al. Chronic pain characteristics and gait in older adults: the MOBILIZE Boston Study II. Arch Phys Med Rehabil. 2020;101:418-425. doi:10.1016/j.apmr.2019.09.010

7. Mickle KJ, Munro BJ, Lord SR, Menz HB, Steele JR. Foot pain, plantar pressures, and falls in older people: a prospective study. $\mathrm{J} \mathrm{Am}$ Geriatr Soc. 2010;58:1936-1940. doi:10.1111/j.1532-5415.2010.03 061.x

8. Stevens JA, Corso PS, Finkelstein EA, Miller TR. The costs of fatal and non-fatal falls among older adults. Inj Prev. 2006;12:290-295. doi:10.1136/ip.2005.011015

9. Leveille SG, Bean J, Bandeen-Roche K, Jones R, Hochberg M, Guralnik JM. Musculoskeletal pain and risk for falls in older disabled women living in the community. $J$ Am Geriatr Soc. 2002; 50:671-678. doi:10.1046/j.1532-5415.2002.50161.x

10. Menz HB, Morris ME, Lord SR. Foot and ankle risk factors for falls in older people: A prospective study. J Gerontol A Biol Sci Med Sci. 2006;61A:866-870. doi:10.1093/gerona/61.8.866

11. Ferrucci L, Guralnik JM, Buchner D, et al. Departures from linearity in the relationship between measures of muscular strength and physical performance of the lower extremities: the Women's Health and Aging Study. J Gerontol A Biol Sci Med Sci. 1997;52:275-285. doi:10.1093/gerona/52A.5.M275

12. Dumurgier J, Elbaz A, Ducimetière P, Tavernier B, Alpérovitch A, Tzourio C. Slow walking speed and cardiovascular death in well functioning older adults: prospective cohort study. BMJ. 2009; 339:4460. doi:10.1136/bmj.b4460 
13. Hausdorff JM. Gait dynamics, fractals and falls: findings meaning in the stride-to-stride fluctuations of human walking. Hum Mov Sci. 2007;26:555-589. doi:10.1016/j.humov.2007.05.003

14. Riskowski JL, Hagedorn TJ, Dufour AB, Hannan MT. Associations of region-specific foot pain and foot biomechanics: the Framingham Foot Study. J Gerontol A Biol Sci Med Sci. 2015;70:1281-1288. doi:10.1093/gerona/glv067

15. Quach L, Galica AM, Jones RN, et al. The nonlinear relationship between gait speed and falls: the MOBILIZE Boston Study. J Am Geriatr Soc. 2011;59:1069-1073. doi:10.1111/j.1532-5415.2011.03408.x

16. Kyrdalen IL, Thingstad P, Sandvik L, Ormstad H. Associations between gait speed and well-known fall risk factors among community-dwelling older adults. Physiother Res Int. 2019; 24:1743. doi: $10.1002 /$ pri.1743

17. Tay MA, Poh M, Lee LH, Ismail NH. Normative gait speed and its relation to falls in the community dwelling elderly in Singapore. Physiother. 2015;101:1493-1494. doi:10.1016/j.physio.2015.03.1471

18. Samah ZA, Nordin NAM, Shahar S, Singh DKA. Can gait speed test be used as a falls risk screening tool in community dwelling older adults? A review. Polish Ann Med. 2016;23:61-67. doi:10.1016/j. poamed.2015.04.007

19. Callisaya ML, Blizzard L, Schmidt MD, et al. Gait, gait variability and the risk of multiple incident falls in older people: a population-based study. Age Aging. 2011;40:481-487. doi:10.1093/ageing/afr055

20. Hausdorff JM, Rios DA, Edelberg HK. Gait variability and fall risk in community-living older adults: a 1-year prospective study. Arch Phys Med Rehabil. 2001;82:1050-1056. doi:10.1053/apmr.2001.24893

21. Beauchet O, Allali G, Annweiler C, et al. Does change in gait while counting backward predict the occurrence of a first fall in older adults? Gerontology. 2008;54:217-223. doi:10.1159/000127318

22. Van Swearingen JM, Paschal KA, Bonino P, Chen TW. Assessing recurrent fall risk of community-dwelling, frail older veterans using specific tests of mobility and the physical performance test of function. J Gerontol A Biol Sci Med Sci. 1998;53:457-464. doi:10. 1093/gerona/53A.6.M457

23. Kasović M, Štefan L, Zvonař M. Domain-specific and total sedentary behavior associated with gait velocity in older adults: the mediating role of physical fitness. Int $J$ Environ Res Public Health. 2020;17:593. doi:10.3390/ijerph17020593
24. Kasović M, Štefan L, Zvonar M. Self-reported vs. measured physical fitness in older women. Clin Interv Aging. 2020;15:425-430. doi:10.2147/CIA.S240156

25. Awale A, Hagedorn TJ, Dufour AB, Menz HB, Casey VA, Hannan MT. Foot function, foot pain, and falls in older adults: the Framingham Foot Study. Gerontology. 2017;63:318-324. doi:10.11 $59 / 000475710$

26. Rosendahl E, Lundin-Olsson L, Kallin K, Jensen J, Gustafson Y, Nyberg L. Prediction of falls among older people in residential care facilities by the Downton index. Aging Clin Exp Res. 2003;15: 142-147. doi:10.1007/BF03324492

27. Van der Leeden M, Dekker JHM, Siemonsma PC, Lek-Westerhof SS, Steultjens MP. Reproducibility of plantar pressure measurements in patients with chronic arthritis: A comparison of one-step, two-step, and three-step protocols and an estimate of the number of measurements required. Foot Ankle Int. 2004;25:739-744. doi:10.1177/ 107110070402501008

28. McHugh ML. Interrater reliability: the kappa statistic. Biochem Med (Zagreb). 2012;22:276-282. doi:10.11613/BM.2012.031

29. Eggermont LH, Penninx BW, Jones RN, Leveille SG. Depressive symptoms, chronic pain, and falls in older community-dwelling adults: the MOBILIZE Boston Study. $J$ Am Geriatr Soc. 2012;60:230-237. doi:10.1111/j.1532-5415.2011.03829.x

30. Henriksen M, Graven - Nielsen T, Aaboe J, Andriacchi TP, Bliddal H. Gait changes in patients with knee osteoarthritis are replicated by experimental knee pain. Arthritis Care Res (Hoboken). 2010;62:501-509. doi:10.1002/acr.20033

31. Sawa R, Doi T, Misu S, et al. The severity and number of musculoskeletal pain associated with gait in community-dwelling elderly individuals. Gait Posture. 2017;54:242-247. doi:10.1016/j.gaitpost. 2017.03.013

32. Cruz-Almeida Y, Rosso A, Marcum Z, et al. Associations of musculoskeletal pain with mobility in older adults: potential cerebral mechanisms. J Gerontol A Biol Sci Med Sci. 2017;72:1270-1276. doi:10.1093/gerona/glx084
Clinical Interventions in Aging

\section{Publish your work in this journal}

Clinical Interventions in Aging is an international, peer-reviewed journal focusing on evidence-based reports on the value or lack thereof of treatments intended to prevent or delay the onset of maladaptive correlates of aging in human beings. This journal is indexed on PubMed Central, MedLine, CAS, Scopus and the Elsevier
Bibliographic databases. The manuscript management system is completely online and includes a very quick and fair peer-review system, which is all easy to use. Visit http://www.dovepress.com/ testimonials.php to read real quotes from published authors. 